Short Report

\title{
Bortezomib Treatment to Potentiate the Anti-tumor Immunity of Ex-vivo Expanded Adoptively Infused Autologous Natural Killer Cells
}

\author{
Andreas Lundqvist ${ }^{1}$, Maria Berg ${ }^{2}$, Aleah Smith ${ }^{2}$, and Richard W. Childs ${ }^{2}{ }^{\bowtie}$ \\ 1. Department of Oncology and Pathology, Cancer Center Karolinska, Karolinska Institutet, Stockholm, Sweden \\ 2. Section of transplantation Immunotherapy, Hematology branch, National Heart, Lung, and Blood Institute, National \\ Institutes of Health, Bethesda, MD 20892, USA
}

$\triangle$ Corresponding author: childsr@mail.nih.gov

(C) Ivyspring International Publisher. This is an open-access article distributed under the terms of the Creative Commons License (http://creativecommons.org/ licenses/by-nc-nd/3.0/). Reproduction is permitted for personal, noncommercial use, provided that the article is in whole, unmodified, and properly cited.

Received: 2011.05.18; Accepted: 2011.06.01; Published: 2011.07.01

\begin{abstract}
Our results show that using an EBV-LCL feeder cell line, large numbers of pure populations of highly activated NK cells can be expanded ex vivo under GMP conditions. With the exception of thyroiditis and constitutional symptoms related to IL-2 therapy, infusions of up to $1 \times 10^{8}$ cells $/ \mathrm{kg}$ of ex vivo expanded NK have been well tolerated and have provided preliminary clinical evidence for mediating anti-tumor immunity in patients with advanced cancer.
\end{abstract}

Key words: Bortezomib, Anti-tumor Immunity, Autologous Natural Killer Cells

Natural killer (NK) cells are innate lymphocytes which have the capacity to kill tumor cells. Unlike antigen specific $\mathrm{T}$ cells, NK cells do not require the presence of a specific tumor antigen for the recognition and killing of cancer cells. NK cell recognition of tumor targets is regulated through a balance of activating and inhibitory signals ${ }^{1}$. Even in the presence of an activating ligand, inhibitory ligands can initiate overriding signals that culminate in a net suppression of NK cell function. The inactivation of NK cells by self-HLA molecules is a potential mechanism by which malignant cells evade host NK cell-mediated immunity. This may limit the ability of both endogenous and adoptively infused autologous NK cells to induce antitumor effects against tumors.

In humans, pilot studies have shown that adoptive transfer of IL-2-activated KIR- incompatible NK cells can be associated with responses in patients with a variety of malignancies 1,2,3. Despite these observations, the therapeutic potential of NK cell-based im- munotherapy remains largely unknown. Adoptively infused allogeneic KIR incompatible NK cells could be used in the setting of an allogeneic HCT as a method to enhance graft-vs-tumor effects against RCC and other cancers 4,5 . However, because mismatched MHC molecules would eventually lead to their rejection by the patient's immune system, outside the setting of an allogeneic transplant, KIR-ligand mismatched NK cells would potentially be of limited therapeutic efficacy. To offset the need for allogeneic KIR incompatible NK cells, we have explored various methods to potentiate autologous NK cell killing of tumor cells.

NK cells lyse tumor targets directly via the perforin/granzyme pathway. They also express Fas ligand and tumor necrosis factor-related apoptosis-inducing ligand (TRAIL), which directly trigger death receptor pathways inducing tumor apoptosis. Recently, we found that the proteosome inhibitor bortezomib sensitized tumors to NK cell killing ${ }^{6}$. Remarkably, NK cells expanded from patients with 
metastatic RCC were significantly more cytotoxic against the patient's own autologous tumor cells when tumors were pretreated with bortezomib compared to untreated tumors. The enhanced NK cell killing effect was found to be dependent on the up-regulation of receptors on the tumor surface for tumor necrosis factor related apoptosis inducing ligand (TRAIL) and to drug-induced augmentation of tumor caspase- 8 activity, leading to enhanced tumor killing via perforin/granzyme mediated NK-tumor cytotoxicity. Tumors sensitized to NK cell cytotoxicity showed a significant increase in surface expression of DR5 (TRAIL-R2) and enhanced susceptibility to NK cell killing was completely abolished by blocking TRAIL on NK cells and partially abolished by blocking DR5 on tumor cells. Remarkably, non-malignant cells including hematopoietic cells and fibroblasts did not show enhanced susceptibility to killing by NK cells or recombinant TRAIL after exposure to either of these agents suggesting that this sensitizing effect may be specific to malignant cells.

Murine studies conducted in our lab have shown that bortezomib also augments the anti-tumor effects of syngeneic NK cell infusions in tumor-bearing animals with this effect being further enhanced by regulatory T-cell (Treg) depletion 7,8 .

Based on these preclinical data, a clinical trial was initiated to explore the safety and antitumor efficacy of escalating doses of adoptively infused ex vivo expanded autologous NK cells following bortezomib treatment in patients with advanced malignancies ${ }^{9}$. Patients age 18-70 with chronic lymphocytic leukemia or a variety of different metastatic tumors with progressive disease refractory to conventional therapy were eligible for study. Patients underwent an apheresis to isolate NK cells that were enriched using immuno-magnetic beads to deplete CD3+ T-cells followed by CD56+ selection. Enriched NK cells were expanded in vitro over 2 weeks using an irradiated clinical grade EBV transformed lymphoblastoid cell line10. Three days prior to NK cell infusion, subjects received a single injection of pentostatin $\left(4 \mathrm{mg} / \mathrm{m}^{2}\right)$ to deplete regulatory T-cells followed by a single injection of bortezomib $\left(1.3 \mathrm{mg} / \mathrm{m}^{2}\right)$ one day prior to the infusion of autologous ex vivo expanded NK cells given on day 0 . To maintain NK cell viability and TRAIL surface expression, 2 million $\mathrm{IU} / \mathrm{m}^{2}$ of IL-2 was given s.c. every 12 hours for 7 days. Patients were restaged at 3 weeks and those with disease regression or stable disease were eligible to receive additional cycles. Cohorts of 3-6 pts have been enrolled into escalating NK cell dose levels $\left(5 \times 10^{6}, 1 \times 10^{7}\right.$, $5 \times 10^{7}$, and $1 \times 10^{8} \mathrm{NK}$ cells $/ \mathrm{kg}$ ). For cohorts $1-4,14$ patients were received a total of $43 \mathrm{NK}$ cell infusions.
Following a 2 week culture, NK cells expanded a median 184 fold (range 58-683) with $42 / 43$ cultures expanding successfully to achieve the target NK cell dose. NK cells harvested a median 14 days after expansion (range 14-16) contained a median 99.7\% (range 96-100) CD3-/CD56+ NK cells, 0\% CD3+ T-cells and had a median $87 \%$ (range 71-93) viability by 7AAD/Annexin V staining.

The most common adverse events related to treatment were attributable to IL-2 therapy and included grades I-II fever, renal insufficiency, edema and hypotension. Two pts developed laboratory evidence for acute thyroiditis with one subsequently developing hypothyroidism requiring thyroid replacement therapy. The best clinical response observed to date has included $7 / 14$ patients having stable disease including two patients who had more than a $30 \%$ decline in serum tumor markers and 1 patient with metastatic kidney cancer who has had a minor response. NK cell cohorts 1-4 have established that a single infusion of autologous NK cells following bortezomib treatment is safe (i.e. a dose limiting toxicity was not reached in cohorts 1-4). Our animal models using sequential bioluminescence imaging to determine tumor burden have shown that maximum anti-tumor effects and prolongation of survival are observed in tumor bearing mice that received repeated infusions of NK cells following bortezomib treatment ${ }^{7}$. Therefore, we added additional cohorts to this ongoing phase I study to evaluate the safety of escalating doses of a second NK cell infusion given with each treatment cycle.

In conclusion, these results show that using an EBV-LCL feeder cell line, large numbers of pure populations of highly activated NK cells can be expanded ex vivo under GMP conditions. With the exception of thyroiditis and constitutional symptoms related to IL-2 therapy, infusions of up to $1 \times 10^{8}$ cells $/ \mathrm{kg}$ of ex vivo expanded NK have been well tolerated and have provided preliminary clinical evidence for mediating anti-tumor immunity in patients with advanced cancer. Once the maximum achievable dose of ex vivo expanded cells that can be infused safely is established, phase II trials in specific tumor subtypes may be initiated to establish overall efficacy of this approach.

\section{Acknowledgments}

This work was supported by the intramural research program of NIH, National Heart, Lung, and Blood Institute, Hematology Branch. We wish to acknowledge the ACKC (Action to Cure Kidney Cancer) for their support of this research as well as the 
Dean R. O'Neill Memorial Fellowship for generous contributions supporting this research.

\section{Conflict of Interest}

The authors have declared that no conflict of interest exists.

\section{References}

1. Srivastava S, Lundqvist A, Childs R. Natural Killer cell immunotherapy for cancer: a new hope. Cytotherapy 2008;10:775-83

2. Miller JS, Soignier Y, Panoskaltsis-Mortari A, et al. Successful adoptive transfer and in vivo expansion of human haploidentical NK cells in patients with cancer. Blood 2005;105:3051-3057

3. Rubnitz JE IH, Ribeiro RC, Pounds S, et al. A pilot study to determine the safety and feasibility of haploidentical natural killer cell transplantation in childhood acute myeloid leukemia. J Clin Oncol 2010;28:955-9

4. Lundqvist A, McCoy JP, Samsel L, et al. Reduction of GVHD and enhanced antitumor effects after adoptive infusion of alloreactive Ly49-mismatched NK cells from MHC-matched donors. Blood 2007;109:3603-6

5. Ruggeri L, Capanni M, Urbani E, et al. Effectiveness of donor natural killer cell alloreactivity in mismatched hematopoietic transplants. Science 2002;295:2097-100

6. Lundqvist A, Abrams SI, Schrump DS, et al. Bortezomib and depsipeptide sensitize tumors to tumor necrosis factor-related apoptosis-inducing ligand: a novel method to potentiate natural killer cell tumor cytotoxicity. Cancer Res 2006;66:7317-25

7. Lundqvist A, Yokoyama H, Smith A, et al. Bortezomib treatment and regulatory $\mathrm{T}$-cell depletion enhance the antitumor effects of adoptively infused NK cells. Blood 2009;113:6120-7

8. Lundqvist A, Su S, Rao S, et al. Cutting edge: bortezomib-treated tumors sensitized to NK cell apoptosis paradoxically acquire resistance to antigen-specific T cells. J Immunol 2010;184:1139-42

9. Lundqvist A, et al. Adoptive Infusion of Ex-Vivo Expanded Autologous Natural Killer (NK) Cells in Cancer Patients Treated with Bortezomib to Sensitize to NK-TRAIL Cytotoxicity. Blood 2010; 114(1564):4080abs

10. Berg M, Lundqvist A, McCoy PJr, et al. Clinical-grade ex vivo-expanded human natural killer cells up-regulate activating receptors and death receptor ligands and have enhanced cytolytic activity against tumor cells. Cytotherapy 2009;11:341-55 